\title{
Improved midterm outcomes for type A (central) pulmonary emboli treated surgically
}

James P. Greelish, MD, Marzia Leacche, MD, Natalia S. Solenkova, MD, PhD, Rashid M. Ahmad, MD, and John G. Byrne, MD

Objectives: We propose a simplified anatomic classification for pulmonary emboli that algorithmically differentiates those who might be best treated with surgical pulmonary embolectomy (type A) from those best treated medically (type B). We hypothesized that patients with type A pulmonary emboli treated with immediate surgical embolectomy demonstrate superior long-term survival compared with patients with type A pulmonary emboli treated medically.

Methods: Patients admitted between 2002 and 2008 with a diagnosis of pulmonary emboli made based on computed tomographic angiographic imaging $(\mathrm{n}=779)$ were analyzed. Computed tomographic angiographic images were reviewed in a blind fashion, and anatomic classification of emboli was made. Patients with central thrombus, defined by location medial to the lateral mediastinal boundaries (ie, involving the main, primary, or both branch pulmonary arteries), were classified as having type A pulmonary emboli $(n=107)$, whereas those with peripheral pulmonary emboli located beyond these boundaries were classified as having type B pulmonary emboli $(\mathrm{n}=672)$. Four patients with type A pulmonary emboli treated with catheter embolectomy were excluded from the analysis.

Results: Of the 103 patients with type A pulmonary emboli, $15(14 \%)$ were treated with immediate surgical pulmonary embolectomy, and $88(85 \%)$ were treated medically. Patients with type A pulmonary emboli treated surgically had similar 30 -day mortality compared with those treated medically $(13 \%$ vs $17 \%, P=.532)$. At a mean of $24 \pm 18$ months' follow-up (range, 1-82 months), survival at 1, 3, and 5 years for patients with type A pulmonary emboli treated surgically was significantly better than that in the patients with type A pulmonary emboli treated medically $(P=.0001)$.

Conclusions: For patients with type A pulmonary emboli, immediate surgical intervention appears to offer superior midterm survival compared with medical treatment alone. Although the medical and surgical groups were substantially different and the differences might have affected survival, this simplified classification for pulmonary emboli might help direct optimal treatment strategies. (J Thorac Cardiovasc Surg 2011;142:1423-9)

Traditionally, pulmonary emboli (PE) have been treated medically with anticoagulation, whereas thrombolysis and surgical embolectomy have been reserved for patients with massive PE with hemodynamic collapse. As a consequence, surgical mortality rates have been discouraging, ranging from $16 \%$ to $64 \% .{ }^{1,2}$ In the late 1990 s, the Brigham and Women's group proposed a more liberal application of surgical embolectomy before hemodynamic shock, resulting in improved short-term and midterm out-

\footnotetext{
From the Department of Cardiac Surgery, Vanderbilt Heart \& Vascular Institute, Nashville, Tenn.

Funding and technical support for this project were provided by the Vanderbilt Heart \& Vascular Institute and the Cardiac Surgery Research Fund of Vanderbilt University Medical Center.

Disclosures: Authors have nothing to disclose with regard to commercial support.

Received for publication May 19, 2010; revisions received Nov 11, 2010; accepted for publication Dec 9, 2010; available ahead of print April 11, 2011.

Address for reprints: John G. Byrne, MD, Vanderbilt University Medical Center, Department of Cardiac Surgery, 1215 21st Ave South, Nashville, TN 37232-8802 (E-mail: john.byrne@ vanderbilt.edu).

$0022-5223 / \$ 36.00$

Copyright (C) 2011 Published by Elsevier Inc. on behalf of The American Association for Thoracic Surgery

doi:10.1016/j.jtcvs.2010.12.030
}

comes. They attributed their results to earlier correction of right ventricular (RV) dysfunction and higher avoidance of long-term RV failure. ${ }^{3}$

For surgical embolectomy to be successful, the embolus must be both anatomically accessible and hemodynamically relevant. Removal of clot beyond the third-order pulmonary artery branches is technically difficult and fraught with the potential risk of vessel laceration. Moreover, clots at this level, given alternate tributaries, are less likely to represent a significant obstruction to flow. Conversely, proximal clot is more easily removed, larger, and more likely to present a significant hemodynamic burden to the right ventricle. Therefore we proposed a simplified anatomic classification system for PE based on location within the pulmonary arterial tree, which algorithmically defines those patients who might benefit from surgical embolectomy versus those in whom medical treatment is ideal.

Patients with central thrombus, defined by location within the lateral mediastinal boundaries (involving the main, primary, or both branch pulmonary arteries) were classified as having type A PE (Figure 1), whereas those beyond these boundaries were classified as having type B PE (Figure 2). 


$$
\begin{aligned}
& \text { Abbreviations and Acronyms } \\
& \mathrm{CI}=\text { confidence interval } \\
& \mathrm{CPR}=\text { cardiopulmonary resuscitation } \\
& \mathrm{CT}=\text { computed tomography } \\
& \mathrm{DVT}=\text { deep venous thrombosis } \\
& \mathrm{PE}=\text { pulmonary emboli } \\
& \mathrm{RV}=\text { right ventricle }
\end{aligned}
$$

We hypothesized that patients with type A PE treated with immediate surgical embolectomy would derive faster relief of RV dysfunction, resulting in superior midterm survival compared with patients with type A PE treated medically. This algorithm is meant to be similar to that used to treat type A and type B aortic dissection. Just as a Stanford type A dissection reflects a condition best treated surgically, so also a Vanderbilt type A PE reflects a surgical condition.

\section{MATERIALS AND METHODS}

We conducted a single-center, retrospective cohort study. Institutional review board approval was obtained to review the medical and imaging records of patients who were admitted to Vanderbilt University Hospital with the diagnosis of PE from January 2002 to December 2008. Those with computed tomographic $(\mathrm{CT})$ angiographic images $(\mathrm{n}=779)$ were then analyzed. Each CT angiographic image was then reviewed retrospectively in a blinded fashion by a board-certified cardiothoracic surgeon, and anatomic classification of emboli was made. Patients with central thrombus, defined by location within the lateral mediastinal boundaries (ie, involving the main, primary, or both branch pulmonary arteries), were classified as having type A PE $(n=107)$, whereas those with thrombus beyond these boundaries were classified as having type B PE $(n=672)$. Four patients with type A PE treated with catheter embolectomy were excluded, leaving 103 patients with type A PE for analysis. All patients with type B PE were treated medically. Patients considered for surgical intervention underwent echocardiographic analysis to assess RV function. RV dysfunction was defined by an $\mathrm{RV} /$ left ventricular end-diastolic diameter ratio of greater than 1 in the apical 4-chamber view, and supportive evidence included paradoxical RV septal systolic motion, loss of inspiratory collapse of the inferior vena cava, and tricuspid regurgitation jet velocity of greater than $2.8 \mathrm{~m} / \mathrm{s}$ or greater than $2.5 \mathrm{~m} / \mathrm{s}$ in the absence of inspiratory collapse of the inferior vena cava.

\section{Treatment Algorithm}

Our proposed treatment algorithm for $\mathrm{PE}$ is presented in Figure 3. In patients with suspected PE, aortic dissection, or both, a contrast chest CT scan will confirm the diagnosis of PE. According to our anatomic classification, patients with a type A PE (central clot) who present with the classic indications for surgical intervention (hemodynamic instability with shock or hypotension, patients with contraindications or inadequate response to thrombolysis, and those with patent foramen ovale and intracardiac thrombi) are treated with surgical pulmonary embolectomy. Patients without the classic indications for surgical intervention are further screened with a 2-dimensional echocardiogram to assess RV function. In the presence of moderate-to-severe RV dysfunction, even without hemodynamic instability, our preferred approach is to treat the patients with immediate surgical pulmonary embolectomy. Patients without RV dysfunction are treated with medical therapy. Patients with type B PE are always treated with medical therapy. Thrombolytics are usually reserved for patients with high-risk PE presenting with cardiogenic shock, persistent arterial hy-

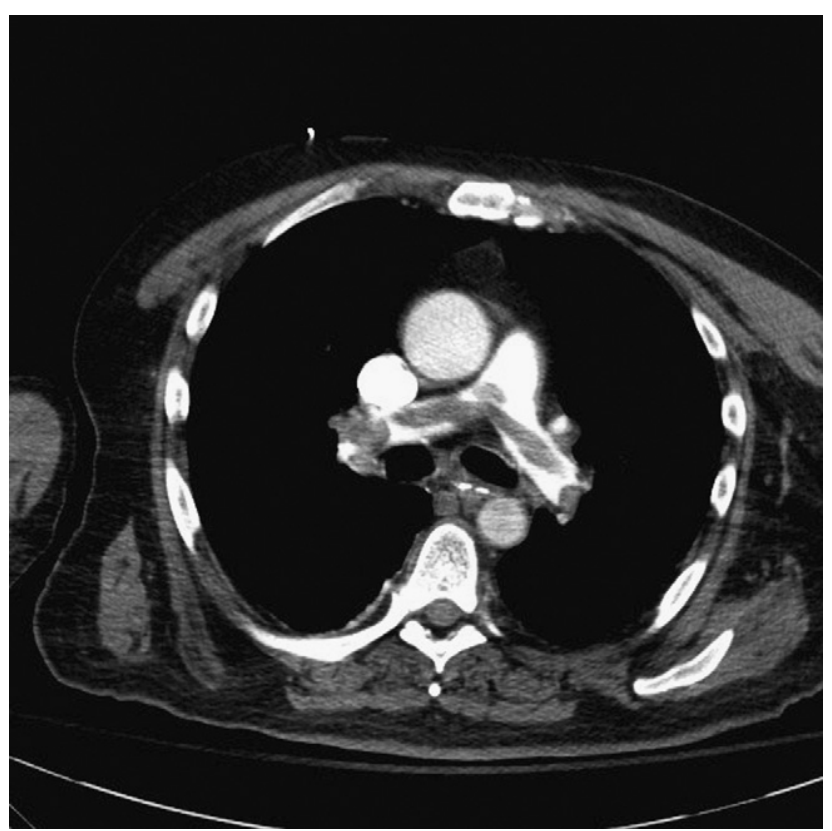

FIGURE 1. Contrast-enhanced chest computed tomographic scan (axial section) showing a large saddle embolus in the pulmonary artery (type A PE).

potension, or both. Routine use of thrombolysis in non-high-risk patients is considered in selected patients with intermediate-risk PE (in whom markers of RV dysfunction and myocardial injury are present).

\section{Surgical Technique}

Our approach to surgical embolectomy has been previously described. ${ }^{3}$ All patients received transesophageal echocardiographic analysis for assessment of right- and left-sided heart function and detection of a patent foramen ovale or atrial septal defect. Median sternotomy with institution of cardiopulmonary bypass was performed. Embolectomies were generally performed on a normothermic beating heart unless cardioplegic arrest was required for closure of a patent foramen ovale or atrial septal defect. A longitudinal incision was made in the main pulmonary arterial trunk toward the left pulmonary artery and retracted with a vein retractor. If necessary for further exposure, an additional arteriotomy was performed on the right pulmonary artery between the aorta and superior vena cava. Thrombus was removed under direct vision with ring forceps and open-tip suction. No Fogarty catheter clot extraction was done to reduce the possibility of injury to the pulmonary artery branches. The pulmonary artery was then closed with 4-0 Prolene sutures, and the patient was weaned from cardiopulmonary bypass. After the operation, all patients received an inferior vena caval filter positioned during fluoroscopy.

\section{Medical Treatment}

Patients treated medically were treated with a combination of enoxaprin (fractionated heparin; Lovenox; Sanofi-Aventis, St Louis, Mo), unfractioned heparin, and warfarin. Recombinant tissue plasminogen activator and alteplase were the 2 thrombolytics used. Patients treated surgically were treated with a combination of heparin (unfractioned) and warfarin.

\section{Statistical Analysis}

The data are expressed as medians and ranges or percentages. For continuous variables, a Mann-Whitney test was used. A 2-tailed Fischer's exact test was used for categorical variables. Long-term survival rates were calculated with the Kaplan-Meier method. We used the STATA 9. for Windows statistical software package (StataCorp, College Station, Tex). 


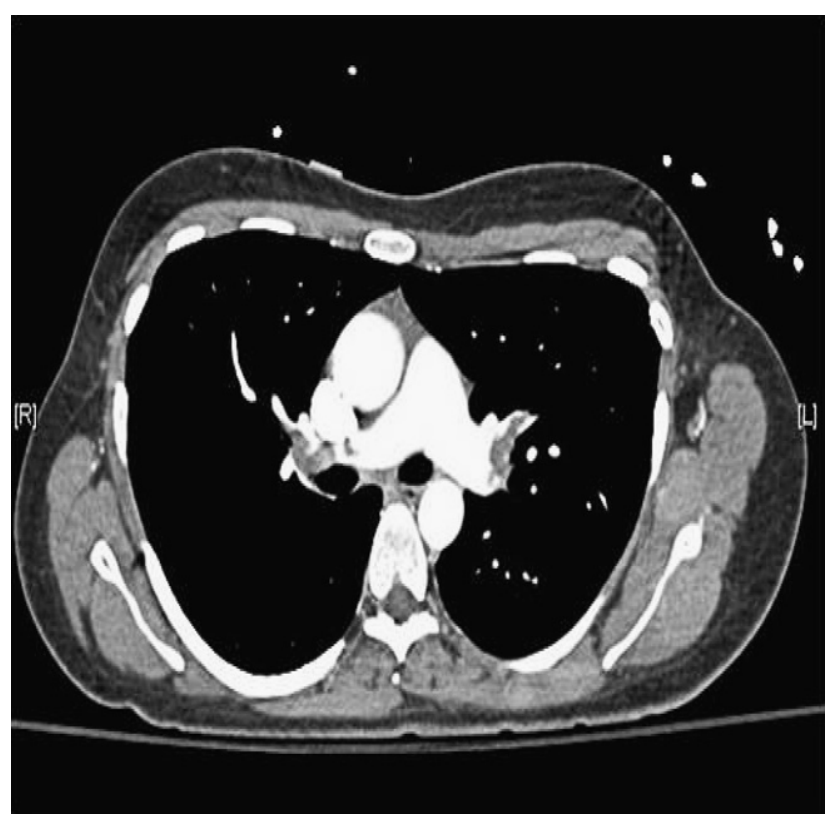

FIGURE 2. Contrast-enhanced chest computed tomographic scan (axial section) showing a large embolus (type B).

\section{RESULTS}

Of the 103 patients with type A PE, 15 (14\%) were treated with immediate surgical pulmonary embolectomy, and $88(85 \%)$ were treated medically. Preoperative pa- tients' demographics and risk factors for deep venous thrombosis (DVT) and PE are shown in Tables 1 and 2. Surgical patients were more likely to be male $(86.7 \%$ vs $63.1 \%, P=.046$ ), have a greater body mass index (31 vs $\left.29 \mathrm{~kg} / \mathrm{m}^{2}, \quad P=.027\right)$, and have a higher baseline creatinine value $(1.2 \mathrm{vs} 1.0 \mathrm{mg} / \mathrm{dL}, P=.004)$. Although symptoms at the time of presentation were also similar between groups, hemodynamic instability defined as a systolic blood pressure of less than $90 \mathrm{~mm} \mathrm{Hg}$ or requiring intravenous pressors was present in nearly half $(47 \%)$ of the surgically treated patients but in only $8 \%$ of the medically treated patients $(P=.001$, Table 3$)$. Thus the surgical population was significantly more ill. In the subgroup of patients who were hemodynamically unstable, the 30-day mortality was similar for patients treated surgically versus patients treated medically $(29 \%$ vs $43 \%$, $P=1.000)$.

At follow-up, the overall mortality (both operative and late) in the medical group was $71 \%$ versus $43 \%$ in the surgical group $(P=.559)$. Consistent with our treatment algorithm, the most common indication to perform pulmonary embolectomy was the presence of RV dysfunction on preoperative echocardiographic analysis $(9 / 15[60 \%])$. Contraindications to thrombolysis were present in only a minority of patients $(4 / 15[27 \%])$ because of recent surgical intervention $(\mathrm{n}=2)$ and recent trauma $(\mathrm{n}=2$, Table 4).

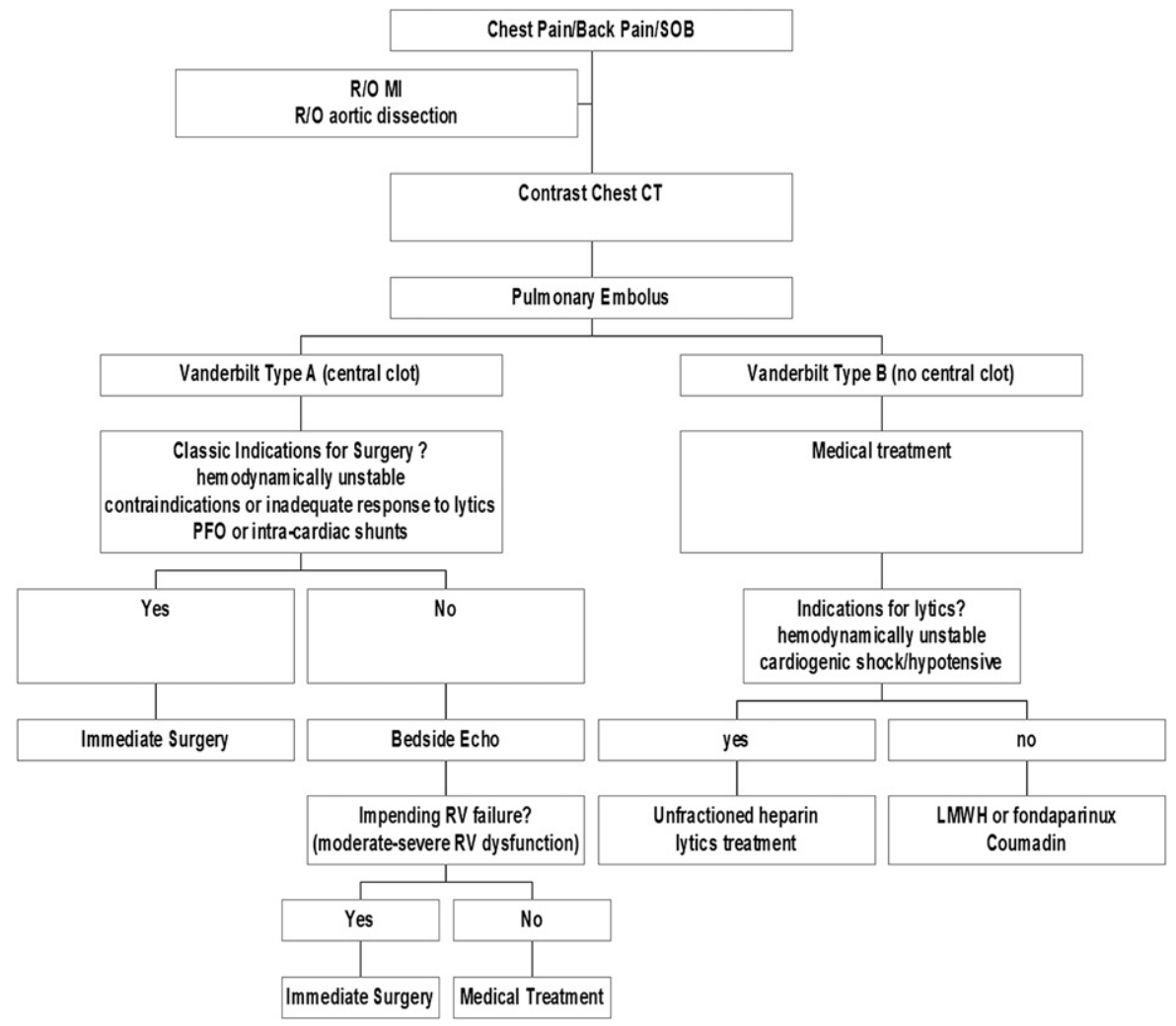

FIGURE 3. Algorithm for diagnosis and treatment of pulmonary emboli. 
TABLE 1. Preoperative data for patients with type A pulmonary emboli

\begin{tabular}{|c|c|c|c|c|}
\hline Variable & Total cohort $(n=103)$ & Surgical $(n=15)$ & Medical $(n=88)$ & $P$ value \\
\hline Median age (y) & $56(18-97)$ & $57(25-90)$ & $56(18-97)$ & .633 \\
\hline Male sex & $62(63.1 \%)$ & $13(86.7 \%)$ & $52(59.1 \%)$ & .046 \\
\hline Median BMI $\left(\mathrm{kg} / \mathrm{m}^{2}\right)$ & $30(16-51)$ & $31(26-49)$ & $29(16-51)$ & .027 \\
\hline Congestive heart failure & $6(6 \%)$ & $2(13 \%)$ & $4(5 \%)$ & .210 \\
\hline Chronic obstructive pulmonary disease & $7(7 \%)$ & $2(13 \%)$ & $5(6 \%)$ & .269 \\
\hline Median baseline creatinine $(\mathrm{mg} / \mathrm{dL})$ & $1.0(0.3-2.6)$ & $1.2(0.7-2.6)$ & $0.9(0.3-1.9)$ & .004 \\
\hline Hypertension & $43(41 \%)$ & $5(33 \%)$ & $37(42 \%)$ & .583 \\
\hline Diabetes & $19(18 \%)$ & $4(27 \%)$ & $15(17 \%)$ & .470 \\
\hline History of myocardial infarction & $1(1 \%)$ & $1(7 \%)$ & $0(0 \%)$ & .146 \\
\hline Previous sternotomy & $4(4 \%)$ & $0(0 \%)$ & $4(5 \%)$ & 1.000 \\
\hline
\end{tabular}

$B M I$, Body mass index.

Eighty percent (12/15) of patients undergoing surgical intervention underwent preoperative echocardiographic analysis, and all (100\%) had RV dysfunction. Echocardiograms were performed in a minority of patients treated medically (35/88 [40\%]), but nearly half (47\%) had RV dysfunction. Therefore RV dysfunction was present in the majority (28/ $47[60 \%])$ of patients with type A PE undergoing echocardiographic analysis. Echocardiography was performed in only a small proportion of patients with type B PE (35/ $672[5 \%]$ ), yet the prevalence of RV dysfunction in those 35 patients was much smaller than in the patients treated surgically ( $10 \%$ vs $60 \%, P=.001)$.

Anticoagulation and inferior vena caval filter strategy also differed significantly between the medically and surgically treated groups. The majority of patients with type A PE treated medically were treated with a combination of enoxaprin (fractionated heparin, 73/88 [83\%]), unfractioned heparin $(18 / 88[20 \%])$, and warfarin $(62 / 88[70 \%])$, whereas less than a third received prophylactic inferior vena caval filter placement (19/88 [21\%]). In contrast, surgical patients were generally treated with heparin (unfractionated, 10/15 [67\%]) and warfarin (9/15 [60\%]) but not fractionated heparin $(0 / 15[0 \%])$. Nearly all surgical patients $(14 / 15[93 \%])$ received inferior vena caval filters (Table 5).

Median cardiopulmonary bypass time was 59 minutes (range, 21-165 minutes). Two (13\%) patients left the operating room with the chest open because of hemodynamic instability. In the postoperative period, $2(13 \%)$ of 15 patients had low cardiac output syndrome, and one of them required RV assist device placement. Postoperative renal failure occurred in $2(13 \%)$ patients, and stroke occurred in $1(7 \%)$ patient. The majority of patients were successfully weaned from the ventilator without the need for tracheostomy $(93 \%)$. There were no reoperations for bleeding.

Despite demonstrating greater comorbidities, with nearly half the patients being hemodynamically unstable or requiring pressors, the patients with type A PE treated surgically had similar 30 -day mortality $(13 \%$ vs $17 \%, P=.532)$

TABLE 2. Risk factors for type A PE

\begin{tabular}{|c|c|c|c|c|}
\hline Variable & Total cohort $(n=103)$ & Surgical $(n=15)$ & Medical $(n=88)$ & $P$ value \\
\hline BMI $>30 \mathrm{~kg} / \mathrm{m}^{2}$ (obesity) & $48(47 \%)$ & $9(60 \%)$ & $39(46 \%)$ & .404 \\
\hline BMI $>40 \mathrm{~kg} / \mathrm{m}^{2}$ (morbid obesity) & $12(12 \%)$ & $4(27 \%)$ & $8(10 \%)$ & .082 \\
\hline History of DVT & $9(9 \%)$ & $2(13 \%)$ & $7(8 \%)$ & .616 \\
\hline Current DVT & $33(32 \%)$ & $2(13 \%)$ & $31(35 \%)$ & .135 \\
\hline Prior PE & $16(16 \%)$ & $2(13 \%)$ & $14(16 \%)$ & 1.000 \\
\hline Smoking history & $25(24 \%)$ & $3(20 \%)$ & $22(25 \%)$ & 1.000 \\
\hline Cancer & $34(33 \%)$ & $2(13 \%)$ & $32(36 \%)$ & .135 \\
\hline Terminal cancer & $21(20 \%)$ & $1(6 \%)$ & $20(22 \%)$ & .295 \\
\hline Hypercoagulability & $8(8 \%)$ & $1(7 \%)$ & $7(8 \%)$ & 1.000 \\
\hline Immobility & $22(21 \%)$ & $4(27 \%)$ & $18(20 \%)$ & .733 \\
\hline Trauma $<1 \mathrm{~m}$ & $3(3 \%)$ & $2(13 \%)$ & $1(1 \%)$ & .055 \\
\hline Major operation $<1 \mathrm{~m}$ & $11(11 \%)$ & $2(13 \%)$ & $9(10 \%)$ & .661 \\
\hline History of CVA & $6(6 \%)$ & $0(0 \%)$ & $6(7 \%)$ & .589 \\
\hline Pregnancy/after delivery & $3(3 \%)$ & $0(0 \%)$ & $3(3 \%)$ & 1.000 \\
\hline Estrogen replacement therapy & $1(1 \%)$ & $0(0 \%)$ & $1(1 \%)$ & 1.000 \\
\hline \multicolumn{5}{|l|}{ Birth control pills } \\
\hline Other & $3(3 \%)$ & $0(0 \%)$ & $3(3 \%)$ & 1.000 \\
\hline Not known & $3(3 \%)$ & $1(7 \%)$ & $2(2 \%)$ & .380 \\
\hline
\end{tabular}

PE, Pulmonary emboli; $B M I$, body mass index; $D V T$, deep venous thrombosis; $C V A$, cerebrovascular accident. 
TABLE 3. Symptoms at presentation for type A pulmonary emboli

\begin{tabular}{|c|c|c|c|c|}
\hline Variable & Total cohort $(n=103)$ & Surgical $(n=15)$ & Medical $(\mathbf{n}=\mathbf{8 8})$ & $P$ value \\
\hline Dyspnea & $86(84 \%)$ & $15(100 \%)$ & $71(81 \%)$ & .070 \\
\hline Chest pain & $49(48 \%)$ & $8(53 \%)$ & $41(47 \%)$ & .781 \\
\hline Hemoptysis & $8(8 \%)$ & $0(0 \%)$ & $8(9 \%)$ & .599 \\
\hline Cough & $21(20 \%)$ & $2(13 \%)$ & $19(22 \%)$ & .730 \\
\hline Tachycardia, HR $>100$ beats $/ \mathrm{min}$ & $41(40 \%)$ & $8(53 \%)$ & $33(38 \%)$ & .267 \\
\hline Hemoinstability ( $\mathrm{SBP}<90 \mathrm{~mm} \mathrm{Hg}$ or requiring pressors) & $14(14 \%)$ & $7(47 \%)$ & $7(8 \%)$ & .001 \\
\hline Syncope & $12(12 \%)$ & $4(28 \%)$ & $8(9 \%)$ & .072 \\
\hline Cardiac arrest & $3(3 \%)$ & $1(7 \%)$ & $2(2 \%)$ & .380 \\
\hline Leg pain/swelling & $20(19 \%)$ & $1(7 \%)$ & $19(22 \%)$ & .292 \\
\hline
\end{tabular}

$H R$, Heart rate; $S B P$, systolic blood pressure.

compared with the medically treated patients. Patients with type B PE had an overall 10\% (53/672) 30-day mortality.

Notably, the 2 deaths that occurred in the surgically treated group were both in patients requiring preoperative cardiopulmonary resuscitation (CPR). The first occurred in a 90-year-old woman of small stature (body mass index, $26.24 \mathrm{~kg} / \mathrm{m}^{2}$ ) who presented with acute respiratory failure requiring intubation. She experienced hypotension leading to pulseless electrical activity arrest and required CPR 3 times before being taken to surgical intervention. A CT scan of the chest revealed a large saddle embolus occluding the main pulmonary and primary branch vessels, with an echocardiogram demonstrating severe RV dysfunction. Emergency salvage pulmonary embolectomy was performed successfully. However, the patient had multisystem organ failure in the postoperative period, and care was withdrawn.

The second death occurred in a 37-year-old morbidly obese man ( $331 \mathrm{lbs}$; body mass index, $43 \mathrm{~kg} / \mathrm{m}^{2}$ ) who presented with marked hemodynamic instability (blood pressure, $80 / 52 \mathrm{~mm} \mathrm{Hg}$; heart rate, 123 beats/min). Echocardiographic analysis revealed a massive right atrial clot extending into the right ventricle and pulmonary trunk. On transfer to the operating room, he arrested, and CPR was initiated with open cardiac message. After being started on cardiopulmonary bypass, successful pulmonary embolectomy was performed, and he was transferred to the intensive care unit. He had severe right-sided heart failure requiring

TABLE 4. Indications for surgical embolectomy in patients with type A PE

\begin{tabular}{ll}
\hline \multicolumn{1}{c}{ Indication } & \multicolumn{1}{c}{ No. $(\mathbf{n}=\mathbf{1 5})$} \\
\hline Contraindication to thrombolysis & $4 / 15(27 \%)$ \\
& $\mathrm{n}=2$, hemodynamically unstable \\
& $\mathrm{n}=2$ with RV dysfunction \\
Large RA-RV thrombus & $2 / 15(13 \%)$ \\
& $\mathrm{n}=1$, left renal cell carcinoma with \\
& caval thrombus extending up to \\
& the RA \\
& $\mathrm{n}=1$, large RA thrombus \\
RV dysfunction & $9 / 15(60 \%)$ \\
\hline
\end{tabular}

$R A$, Right atrium; $R V$, right ventricle.
RV assist device placement but went on to have multisystem organ failure and died on postoperative day 7 .

Among the patients treated medically, 17 died within 30 days, and $8(47 \%)$ of 17 had cardiopulmonary failure caused by PE.

Kaplan-Meier survival analysis (Figure 4) revealed improved long-term outcomes in the surgical group. Mean follow-up was $24 \pm 18$ months (range, 1-82 months), and follow-up was $100 \%$ complete. Actuarial survival was $92 \% \pm 7.4 \%$ ( $95 \%$ confidence interval [CI], $0.57 \%$ to $0.98 \%$ ) for patients with type A PE treated surgically at 1 and 3 years versus $74 \% \pm 5 \%(95 \% \mathrm{CI}, 0.62 \%$ to $0.82 \%$ ) and $66.6 \% \pm 6.1 \%$ (95\% CI, $0.53 \%$ to $0.77 \%$ ) for patients with type A PE treated medically $(P=.0001)$, respectively.

Regarding late mortality, the cause of death in the surgical group was cancer (1 patient), and for the medically treated group $(\mathrm{n}=23)$, the causes were cancer $(14 / 23$ $[60 \%])$, cardiac $(3 / 23[13 \%])$, neurologic $(3 / 23[13 \%])$, respiratory failure $(1 / 23[4 \%])$, and "unknown" $(3 / 23$ $[13 \%])$.

\section{DISCUSSION}

In this series of patients with a documented large central clot burden (type A) have similar 30-day mortality with medical or surgical treatment, despite the latter group being more hemodynamically unstable and with the presence of RV dysfunction. All patients in this series and 2 of 3 in our Boston series ${ }^{3}$ who required preoperative CPR died. Had we not operated on the 2 patients who required preoperative CPR, the operative mortality in the surgical group would have been $0 \%$. This suggests that preoperative CPR might be a contraindication to surgical intervention. The late survival, however, seems better for patients with type A PE treated surgically compared with those treated medically. Thus surgical pulmonary embolectomy should be considered for patients with documented large central clot burden (type A) and documented impending RV failure.

Over the past few decades, there has been a significant decrease in mortality from cardiovascular disease, the 
TABLE 5. Treatment of type A pulmonary emboli

\begin{tabular}{|c|c|c|c|c|}
\hline Variable & Total cohort $(n=103)$ & Surgical $(n=15)$ & Medical $(\mathbf{n}=\mathbf{8 8})$ & $P$ value \\
\hline Inferior vena caval filter & $33(32 \%)$ & $14(93 \%)$ & $19(21 \%)$ & .001 \\
\hline Preexisting & $4 / 34$ & $0 / 14$ & $4 / 19$ & \\
\hline In addition to medical/surgical treatment & $29 / 34$ & $14 / 14$ & $15 / 19$ & \\
\hline Enoxaprin (Lovenox) & $73(71 \%)$ & $0(0 \%)$ & $73(83 \%)$ & .001 \\
\hline Other heparins & $28(27 \%)$ & $10(67 \%)$ & $18(20 \%)$ & .001 \\
\hline Warfarin sodium (Coumadin) & $71(69 \%)$ & $9(60 \%)$ & $62(70 \%)$ & .547 \\
\hline Aspirin & $21(20 \%)$ & $11(73 \%)$ & $10(11 \%)$ & .001 \\
\hline Clopidogrel (Plavix) & $3(3 \%)$ & $2(13 \%)$ & $1(1 \%)$ & .055 \\
\hline Thrombolytics & $2(2 \%)$ & & $2(2 \%)^{*}$ & \\
\hline
\end{tabular}

*Thrombolytics used: 1 patient, alteplase; 1 patient, recombinant tissue plasminogen activator.

leading cause of death in the United States. Despite this, mortality from DVT and PE has remained high $(17 \%$ at 3 months). ${ }^{4}$ Current estimates indicate that 350,000 to 600,000 persons per year are given diagnoses of DVT and PE in the United States, with 100,000 to 180,000 deaths attributed to these causes annually. The significance of the high mortality associated with this disease has led the US Surgeon General in 2008 to issue a call to action for the prevention of DVT and PE to bring national attention to this issue. ${ }^{5}$

In a multivariate analysis of a large cohort of patients $(n=2454)$ with PE in the International Cooperative Pulmonary Embolism Registry, RV hypokinesis was found to be the most significant prognostic factor, doubling the mortality rate when present. ${ }^{4}$ During the first 30 days after PE, acute RV failure accounts for the majority of deaths, whereas underlying disease is responsible for mortality thereafter. The right ventricle is a thin-walled structure with poor ability to adapt to acute changes in afterload. Proximal clot in the main pulmonary trunk obstructs flow, induces a pressure load on the right ventricle, and leads to acute RV distension and eventual failure. This study categorized patients with proximal pulmonary artery

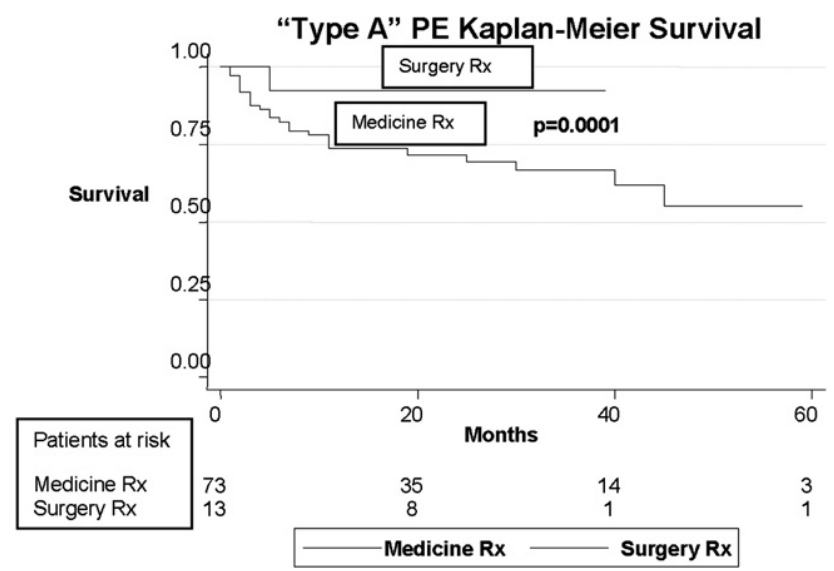

FIGURE 4. Kaplan-Meier survival curve after surgical pulmonary embolectomy and medical treatment for patients with type A pulmonary emboli. clots as having type A disease, thus creating an anatomic surrogate for RV dysfunction. Moreover, classifying patients in this way defines those who have surgically accessible clot and rapidly allows the practitioner to distinguish patients most likely to benefit from surgical intervention. The proposed classification system is analogous to that of the Stanford classification system in aortic dissection in which patients with type A disease carry the highest predicted mortality and require surgical intervention, whereas patients with type B disease can safely be treated medically.

Analysis of preoperative presentation in our study revealed that nearly half of the patients with type A PE treated surgically were hemodynamically unstable compared with only $8 \%$ of those treated medically. This finding reflects adherence to more traditional indications to refer to surgical intervention late and importantly speaks to the higher predicted mortality in the surgically treated subgroup. Mortality rates as high as $58 \%$ versus $15 \%$ have been reported in hemodynamically unstable versus hemodynamically stable patients, respectively, in the International Cooperative Pulmonary Embolism Registry. ${ }^{4}$ As such, the surgical subgroup in our study constituted a much sicker population.

Although additional studies are needed, we believe the success of our treatment protocol stems from its targeting the major causes of morbidity and mortality in PE, namely RV failure, recurrent PE, untreated additional clots, and chronic thromboembolic disease leading to chronic pulmonary hypertension. Surgical pulmonary embolectomy provides rapid relief of RV dysfunction by means of removal of clot early in the pathogenic process. As such, RV distension and strain are minimized. Embolectomy also prevents clot organization and thromboembolic scarring, lessening the risk of long-term pulmonary hypertension. Ninetythree percent of patients treated surgically had intraoperatively placed inferior vena caval filters. Although seemingly an aggressive practice compared with most published guidelines for inferior vena caval filter placement, large natural history studies have shown that that nearly half $(47 \%)$ of those with recurrent PE (17\%) die within 3 months. ${ }^{4,6}$ Moreover, inferior vena caval filter placement in cases of 
massive PE has been shown to reduce recurrent PE and 90day mortality. ${ }^{7}$ Therefore it is our opinion that recurrent PE remains a significant threat after a massive pulmonary embolism and warrants inferior vena caval filter placement.

Importantly, RV dysfunction at the time of presentation has been shown to be the most important determinant of early mortality in patients with PE. ${ }^{4,8,9}$ Consequently, echocardiograms were obtained for the majority of patients treated surgically $(80 \%)$, which is consistent with our planned treatment algorithm. Three patients did not receive preoperative echocardiographic analysis because their conditions were unstable, but all of them underwent intraoperative transesophageal echocardiographic assessment. In contrast, only $40 \%$ of patients in the medically treated group underwent echocardiographic analysis. RV dysfunction was found in all surgical patients and $47 \%$ of medically treated patients. Therefore RV failure was present in the majority of patients with type A PE (surgical and medical) with echocardiograms. This finding is consistent with our hypothesis that our anatomic classification system serves as a surrogate marker for RV dysfunction.

Because of inconsistent treatment algorithms over a 6-year period, some patients treated medically were simply never considered for surgical intervention. Currently, we have a team approach, with surgeons and cardiologists working together as a team and providing the same algorithm of treatment. Moreover, only $40 \%$ of patients in the medical group received echocardiographic analysis of the right ventricle. These observations point to the need for a universally accepted approach to patients presenting with PE. We believe that all patients with suspected PE should undergo imaging (CT angiographic imaging) to confirm the diagnosis and allow anatomic classification as type A or type B. In addition, all patients should undergo echocardiographic analysis to assess RV function. Patients with type A PE should be referred for consideration of surgical embolectomy because our results have shown that the majority of these patients $(29 / 47$ [62\%]) will have significant $\mathrm{RV}$ dysfunction, the main determinant of mortality in the first 30 days. Expeditious embolectomy in patients with type A PE with RV dysfunction will subsequently result in improved long-term survival. We do not recommend catheter embolectomy because of the risk of PE fragmentation.

\section{Limitations}

This is a retrospective review in a relatively small cohort. Verification of these results in a randomized trial would be optimal. The type of treatment was based on individual patient selection. Although not statistically significant, cancer occurred more frequently in the medically treated patients than in those treated surgically. Consequently, it is possible that survival statistics were affected accordingly. Also, a more aggressive anticoagulation and inferior vena caval filter strategy was used in the surgical group.

\section{CONCLUSIONS}

For type A PE, immediate surgical intervention offers superior long-term survival compared with medical treatment alone. Immediate surgical embolectomy should be strongly considered in this group of patients because most have RV dysfunction, the most important determinant of early mortality. This new classification for PE simplifies decision making and might help direct optimal treatment strategies.

Other contributing authors included Tarek S. Absi, MD; Stephen K. Ball, MD; Jorge M. Balaguer, MD; Julian S. Bick, MD; Susan S. Eagle, MD; Pretorius Mias, MD; Robert J. Deegan, MD; Steven J. Hoff, MD; Michael R. Petracek, MD; and AnneMarie Thompson, MD

\section{References}

1. Gray HH, Morgan JM, Paneth M, et al. Pulmonary embolectomy for acute massive pulmonary embolism: an analysis of 71 cases. Br Heart J. 1988;60:196-200.

2. Meyer G, Tamisier D, Sors H, et al. Pulmonary embolectomy: a 20-year experience at one center. Ann Thorac Surg. 1991;51:232-6.

3. Leacche M, Unic D, Goldhaber SZ, et al. Modern surgical treatment of massive pulmonary embolism: results in 47 consecutive patients after rapid diagnosis and aggressive surgical approach. J Thorac Cardiovasc Surg. 2005;129:1018-23.

4. Goldhaber SZ, Visani L, De Rosa M. Acute pulmonary embolism: clinical outcomes in the International Cooperative Pulmonary Embolism Registry (ICOPER). Lancet. 1999;353:1386-9.

5. US Department of Health and Human Services. The surgeon general's call to action to prevent deep vein thrombosis and pulmonary embolism. Rockville, Md: Department of Health and Human Services; 2008.

6. Buller HR, Agnelli G, Hull RD, et al. Antithrombotic therapy for venous thromboembolic disease: the Seventh ACCP Conference on Antithrombotic and Thrombolytic Therapy. Chest. 2004;126:401S-28.

7. Kucher N, Rossi E, De Rosa M, et al. Massive pulmonary embolism. Circulation. 2006;113:577-82.

8. Ribeiro A, Lindmarker P, Juhlin-Dannfelt A, et al. Echocardiography Doppler in pulmonary embolism: right ventricular dysfunction as a predictor of mortality rate. Am Heart J. 1997; 134:479-87.

9. Kasper W, Konstantinides S, Geibel A, et al. Prognostic significance of right ventricular afterload stress detected by echocardiography in patients with clinically suspected pulmonary embolism. Heart. 1997;77:346-9. 